\title{
Inhibition of BCNU-Resistance in Glioma Cell Lines by Small Interfering Rna Targeting to Interleukin-8 Gene
}

\author{
Si-Ming Xie ${ }^{1}$, Hui Guo ${ }^{2}$, Yong-Rong Yan ${ }^{1}$ and Xue-Yun Zhong ${ }^{1}$ \\ 1. School of Medicine, Jinan University, Guangzhou City, Guangdong 510632, China \\ 2. Postdoctoral station of Clinical Medicine, Jinan University, Guangzhou, Guangdong 510632, China
}

\begin{abstract}
It had been found that interleukin-8 (IL-8) was associated with drug resistance. We previously demonstrated that the resistance to 1, 3-bis (2-chloroethyl)-1-nitrosourea (BCNU) of glioma cell line SWOZ1 was much higher than that of cell line SOWZ2, which were both cloned from the same parental glioma cell line SWO38. In this study, IL-8 was found to be upregulated both in SWOZ1 and SWOZ2-BCNU, a BCNU-resistant glioma cell line. To further investigate the function of IL-8, the BCNU-resistant cell lines SWOZ1 and SWOZ2-BCNU were treated with siRNAs targeting IL-8. The results of quantitative RT-PCR showed that a decreased level of IL-8 mRNA expression in SWOZ1 and SWOZ2-BCNU for more than $90 \%$ compared to negative control and was confirmed by western blot assay $(P<0.05)$ after treated by siRNAs targeting IL-8. Subsequently, the cytotoxicity of BCNU to these cell lines was detected using the cell counting kit-8 assay. As a result, the BCNU resistance was reversed for about $50 \%$ both in these two cell lines $(P<0.05)$. Our data demonstrated that inhibition of IL-8 with specific siRNAs can reverse the BCNU resistant phenotype in glioma cell lines, indicating that IL-8 may play an important role in BCNU-resistance in glioma.
\end{abstract}

Key words: Glioma, IL-8, siRNA, drug resistance, BCNU.

\section{Introduction}

Recent decades have seen only limited progress in treatment trials and basic research on human glioma, the most common central nervous malignancy [1]. The standard therapy for malignant gliomas involves surgical resection, radiotherapy and chemotherapy, with 1, 3-bis (2-chloroethyl)-1-nitrosourea (BCNU) as the most commonly used chemotherapeutic agents $[2,3]$. As a result of the rapid growth and infiltrative spread within the brain, malignant gliomas are generally resistant to all conventional therapies, including surgery, radiation and chemotherapy, and patients have median survivals of only 1-3 years. At the present time, intrinsic or acquired resistance to cytotoxic agents remains the greatest obstacle to the successful treatment of human glioma. Identifying and understanding the diverse mechanisms of

Corresponding Author: Xue-Yun Zhong, Ph.D., professor, research field: pathology. Email: tzxy@jnu.edu.cn. chemoresistance operating in human tumors and developing effective strategies to overcome resistance is the focus of ongoing preclinical and clinical research. In our previous study, we had found that the BCNU resistance to a glioma cell line SWOZ1 was much higher than that to cell line SOWZ2, which were both originated from the same parental glioma cell line SOW38. Subsequently, gene expression was detected by probesets on the Affymetrix U133 plus 2.0 arrays in cell lines SWOZ1, SWOZ2 and SWOZ2- BCNU. Interestingly, interleukin-8 (IL-8; CXCL-8) was found to be elevated both in BCNU resistant cell lines SWOZ1 and SWOZ2-BCNU. It had been found that IL-8 is associated with drug resistance, but few data had shown the correlation of IL-8 with BCNU resistance in glioma. In this study, the function of IL-8 in $\mathrm{BCNU}$ resistance was investigated using small interference RNAs (siRNAs) targeting IL-8 gene in glioma cell lines resistant to BCNU. 


\section{Materials and Methods}

\subsection{Cell Lines and Cell Culture}

The human glioma cell lines SWOZ1 and SOWZ2, and BCNU-esistant cell line SWOZ2 (SWOZ2-BCNU) were incubated in RPMI-1640 medium supplemented with $10 \%$ fetal calf serum (Hyclone, Logan, UT) and 1\% L-glutamine [4]. The BCNU-esistant SWOZ2 (SWOZ2-BCNU) was obtained by continuously culturing the drug-sensitive parental cell line SWOZ2 in medium containing large dose of BCNU $(20 \mu \mathrm{g} / \mathrm{mL})$ for over one year. All cells were cultured at $37{ }^{\circ} \mathrm{C}$ in a humidified incubator with $5 \% \mathrm{CO}_{2}$. To avoid the influence of BCNU, SWOZ2-BCNU was cultured in drug-free medium for over a month.

\subsection{Cytotoxicity Assays and Cell Viability Assay by Cck-8}

Cell counting kit (CCK-8) assay (Dojindo Laboratories, Kumamoto, Japan) was used to detect the cytotoxicity of BCNU in cells and cell viability. For cytotoxicity assays, cells were seeded into 96-well plates at a density of $5 \times 10^{3}$ cells per well. Various gradient concentration of BCNU (Tianjin Drug Factory, Tianjin, China) ranging from $2-128 \mu \mathrm{g} / \mathrm{mL}$ was added to each well $24 \mathrm{~h}$ after seeding. Wells containing no drugs were used for control cell viability and wells containing no cells for blanking. After $48 \mathrm{~h}$ of incubation under normal culture condition, $10 \mu \mathrm{L}$ of CCK-8 solution was added to each well. Two hours later, the optical density (OD) value at $450 \mathrm{~nm}$ wavelength was measured by the microplate reader (Bio Rad, rcules, CA). The 50\% inhibitory concentration $\left(\mathrm{IC}_{50}\right)$, defined as the concentration of the drug that inhibited growth by $50 \%$, was calculated by probit analysis according to the relative absorbance of CCK-8. The resistance index (RI) was calculated by dividing the $\mathrm{IC}_{50}$ of the resistant cell line by that of the parental cell line [5]. All experiments were performed in triplicate.

For cell viability assays, cells were seeded into seven 96-well plates at a density of $1 \times 10^{3}$ cells per well. Wells containing no cells were used as a blank control. CCK-8 assay was done daily from the second to the eighth day of incubation. The OD value of each well was measured as described above. Independent experiments were done in triplicate. Growth curves were drawn based on these OD values [6].

\subsection{Design and Treatment with Sirnas Targeting Il-8}

Three different specific sequences targeting IL-8 were selected according to online siRNA tools of Invitrogen using the IL-8 reference sequence (Gene Bank Accession No. NM_000584.2). The target sequences of si-IL8-1 (5'-GCCAAGGAGTGCTAAAGAA-3'), si-IL8-2 (5'-GCGCCAACACAGAAATTAT-3') and si-IL8-3 (5'-CAAAGAACTGAGAGTGATT-3') are homologous to nt 176-194, 248-266 and 283-301 of the IL-8 specific mRNA, respectively, with invalid RNAi sequence (5'-GCAGGAGCTAT GCTACCATCA-3') as negative control (NC). SiRNAs were synthesized by Ruibo Biotechnology Co. (Guangzhou, China). Transfection of siRNA into cell lines SWOZ1 and SWOZ2-BCNU was performed using lipofectamine 2,000 (Invitrogen, Carlsbad, CA) according to the manufacturer's instructions. $24 \mathrm{~h}$ after transfection, mRNAs of these cells were isolated, and cytotoxicity of BCNU in these cells and cellular proliferation were detected. $48 \mathrm{~h}$ after transfection, protein of these cells was prepared for western blot assay.

\subsection{Analysis of IL-8 mRNA Levels by Quantitative $R T-P C R$}

Total RNA was isolated using Trizol reagent (Invitrogen, Carlsbad, CA) according to the manufacturer's instructions. Quantitative RT-PCR was carried out using a Chromo4 instrument (Bio Rad, rcules, CA) and SYBR ${ }^{\circledR}$ Premix Ex Taq ${ }^{\mathrm{TM}}$ kit (Takara bio, Otsu, Japan) to detect the mRNA level of IL-8, with GAPDH as a normalizing control. The specific 
PCR primer sequences of these genes designed by Primer premier 5.0 software were as follows: IL-8 forward: 5'-TGCCAAGGAGTGCTAAA-3'; IL-8 reverse: 5'- CCCTACAACAGACCCAC-3'; GAPDH forward: 5'-GGATTTGGTCGTATTGGG-3'; GAPDH reverse: 5'-TCGCTCCTGGAAGATGG-3'. Cycling conditions were carried out as: $95^{\circ} \mathrm{C}$ for $10 \mathrm{~m}$ to activate DNA polymerase, proceeding with 40 cycles of $95{ }^{\circ} \mathrm{C}$ for $15 \mathrm{~s}, 55{ }^{\circ} \mathrm{C}$ for $20 \mathrm{~s}$, and $72{ }^{\circ} \mathrm{C}$ for 10 s. Specificity of amplification product was confirmed by melting curve analysis. Independent experiments were done in triplicate. The cycle threshold $(\mathrm{Ct})$ represents a positive PCR result, and is defined as the cycle number at which a sample's fluorescence crossed the threshold automatically determined by the Chromo4 system. The relative changes in gene expression were calculated with the $2^{-\Delta \Delta C t}$ method, where $\Delta C t=C t$ (target gene) $-\mathrm{Ct}$ $(\mathrm{GAPDH})$, and $\Delta \Delta C t=\Delta C t$ (sample) $-\Delta C t$ (calibrator) $[7,8]$. Here sample refers to SWOZ1, SWOZ1/SiIL8 or SWOZ2-BCNU/ SiIL8 cell lines, while calibrator refers to cell line SWOZ2, SWOZ1/Si-NC or SWOZ2-BCNU/ Si-NC. The interferencing efficiency was calculated by the following formula: interferencing efficiency $=\left(1-1 / 2^{-\Delta \Delta C t}\right) \%$.

\subsection{Analysis of Il-8 Protein Levels by Western Blot}

Protein samples were prepared with lysis buffer containing $50 \mathrm{mmol}$ Tris (pH 8.0), $150 \mathrm{mmol} \mathrm{NaCl}, 1 \%$ NP-40, 0.1\% SDS, $100 \mathrm{mg} / \mathrm{mL}$ phenylmethylsulfonyl fluoride (PMSF) and $1 \mathrm{mg} / \mathrm{mL}$ aprotinin. Western blot assay was performed as described previously [9]. Mouse anti-IL-8 (Santa Cruz Biotechnology, CA, USA) at a dilution of 1:1,000 was used to detect the IL-8 protein level. Rabbit anti-GAPDH (Bioworld Technology, Minneapolis, USA) at a dilution of 1:1,000 was used to normalize the quantity of the protein on the blot.

\subsection{Statistic Analysis}

All experiments were repeated at least three times. The data of quantitative RT-PCR and $\mathrm{IC}_{50}$ were expressed as mean \pm standard deviation (SD) value. Statistical analysis for these data was carried out by one-way ANOVA in statistical package SPSS 13.0. All $P$ values $<0.05$ were considered statistically significant.

\section{Results}

3.1 Resistance to BCNU was Enhanced in BCNU-Resistant Cell Line SWOZ1 and SWOZ2-BCNU Compared to SWOZ2

IC50 value in cell line SWOZ1 and SWOZ2-BCNU was $17.1 \mu \mathrm{g} / \mathrm{mL}$ and $18.4 \mu \mathrm{g} / \mathrm{mL}$ respectively, which was significantly higher than that in cell line SWOZ2 (3.5 $\mu \mathrm{g} / \mathrm{mL}, P<0.05$ ). The RI (resistant index) of SWOZ1 and SWOZ2-BCNU was 4.9 and 5.3 respectively. That is to say, the resistance of cell line SWOZ1 and SWOZ2-BCNU against BCNU was 4.9-fold and 5.3-fold higher than that of cell line SWOZ2.

3.2 Expression of IL-8 mRNA and Protein was Overexpressed in BCNU-Resistant Cell Lines SWOZ1 and SWOZ2-BCNU Compared to SWOZ2

IL-8 mRNA level in cell line SWOZ1 and SWOZ2-BCNU was $(6.4 \pm 1.6)$ folds and $(4.0 \pm 0.9)$ folds higher than that in cell line SWOZ2, respectively (Fig. 1a). The result of western blot showed that IL-8 protein expression in cell lines SWOZ1 and SWOZ2-BCNU was $(1.6 \pm 0.2)$-fold and $(2.1 \pm$ $0.2)$-fold higher than that in cell line $\operatorname{SWOZ2}(P<$ 0.05), respectively (Fig. 1b). These results confirmed that the protein expression of IL-8 was significantly overexpressed in cell lines SWOZ1 and SWOZ2-BCNU compared to cell line SWOZ2.

\subsection{Expression of IL-8 mRNA and Protein in Cell Lines} SWOZ1 and SWOZ2-BCNU was Inhibited after Transfection of siRNAs Targeting IL-8

Three different siRNA constructs, siIL8-1, siIL8-2 and siIL8-3, were used to inhibit the mRNA expression of IL-8 in SWOZ1 and SWOZ2-BCNU cell lines, with 
a negative construct as control (NC). As a result, a decreased level of IL-8 mRNA expression in all cell lines treated with siRNAs for more than 60\% compared to negative control was found. Meanwhile, silL8-1 showed the most efficiency of RNAi for more than $90 \%$ both in cell lines SWOZ1 and SWOZ2-BCNU (Fig. 2a), and was selected for RNAi in this study.

Western blot assay was used to detect the IL-8 protein expression after these cell lines were treated by silL8-1. As shown in Fig. 2b, after treatment with siIL8-1 for $48 \mathrm{~h}$, IL-8 protein expression in both cell lines SWOZ1/ siIL8-1 and SWOZ2-BCNU/ siIL8-1 was significantly decreased compared to negative control $(P<0.05)$. After analyzed by IPP software, IL-8 protein level in cell line SWOZ1/siIL8-1 and SWOZ2-BCNU /siIL8-1 was decreased by $(75 \pm 2) \%$ and $(78 \pm 2) \%$ compared to negative control, respectively.

3.4 The BCNU Resistance of Cell Line SWOZ1 and SWOZ2-BCNU was Reversed by siRNAs Targeting
$I L-8$

The cell viability of SWOZ1/siNC, SWOZ1/siIL8-1, SWOZ2-BCNU/siNC and SWOZ2-BCNU/siIL8-1 was measured by a continuous 7-day CCK-8 assay, and growth curves were made according to these OD values alterations of CCK-8 assay. As shown in Fig. 3a, no significant difference was found between the cellular growth of these cell lines $(P>0.05)$. Later, the resistance of these cell lines against BCNU was determined by CCK-8 assay. As shown in Fig. 3b, treatment of BCNU-resistant cell lines SWOZ1 and SWOZ2-BCNU by siRNA targeting IL-8 reversed the BCNU- resistant phenotype, in terms of IC50 values, for about 50\% compared to control cells $(P<0.05)$. The IC50 value was decreased from $16.5 \mu \mathrm{g} / \mathrm{mL}$ in SWOZ1/siNC to $6.7 \mu \mathrm{g} / \mathrm{mL}$ in SWOZ1/si IL8-1, and from $16.7 \mu \mathrm{g} / \mathrm{mL}$ in SWOZ2-BCNU/siNC to $9.5 \mu \mathrm{g} / \mathrm{mL}$ in SWOZ2- BCNU/silL8-1.

(a)

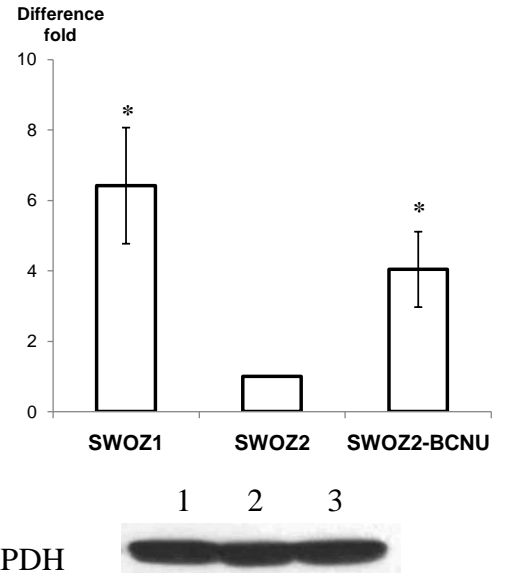

IL-8

Fig. 1 Expression level of IL-8 in cell lines SWOZ1, SWOZ2 and SWOZ2-BCNU.

(a): Comparison of IL-8 mRNA expression in three cell lines. The relative changes in gene expression were calculated with $2^{-4 \Delta C t}$ method by quantitative RT-PCR, normalized against GAPDH. The expression of IL-8 in SWOZ2 was considered a value of one for each individual experiment. *, $P<0.05$ vs. SWOZ2; $n=3$.

(b): Protein expression levels of IL-8 in three cell lines. 1: SWOZ1; 2: SWOZ2; 3: SWOZ2-BCNU. IL-8 protein was detected by western blot, normalized against GAPDH protein; $n=3$. Image-Pro Plus software was used to analyze the result. 


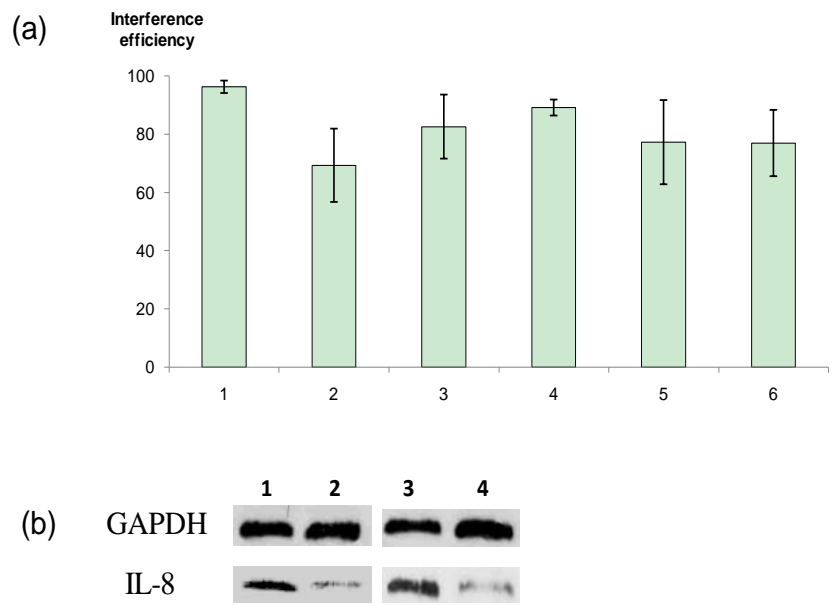

Fig. 2 Modulation of IL-8 expression after RNAi targeting IL-8 in cell lines SWOZ1 and SWOZ2-BCNU.

(a): Interferencing efficiency of IL-8 after siRNAs treatment. Three different siRNA constructs, siIL8-1, siIL8-2 and siIL8-3, were used. Quantitative real-time RT-PCR experiments were performed to evaluate the interferencing efficiency which was calculated by the formula $\left(1-1 / 2^{-\triangle A C t}\right) \%$, normalized against GAPDH.

1: SWOZ1 cells treated with siIL8-1; 2 SWOZ1 cells treated with siIL8-2; 3: SWOZ1 cells treated with siIL8-3; 4 SWOZ2-BCNU cells treated with siIL8-1; 5: SWOZ2-BCNU cells treated with siIL8-2; 6: SWOZ2-BCNU cells treated with siIL8-3; $n=3$.

(b): Modulation of IL-8 protein expression after silL8-1 was transfected into SWOZ1 and SWOZ2-BCNU cells, with a negative construct as control (NC). 1: SWOZ1/siNC; 2: SWOZ1/siIL8-1; 3: SWOZ2-BCNU/siNC; 4: SWOZ2-BCNU/silL8-1. Image-Pro Plus software was used to analyze the result; $n=3$.

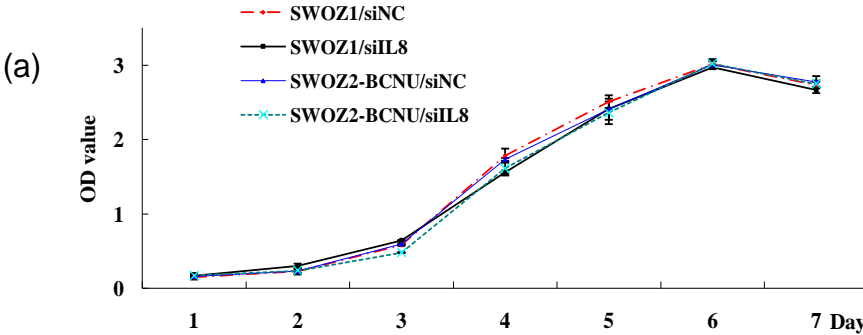

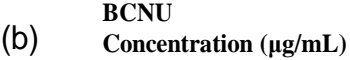

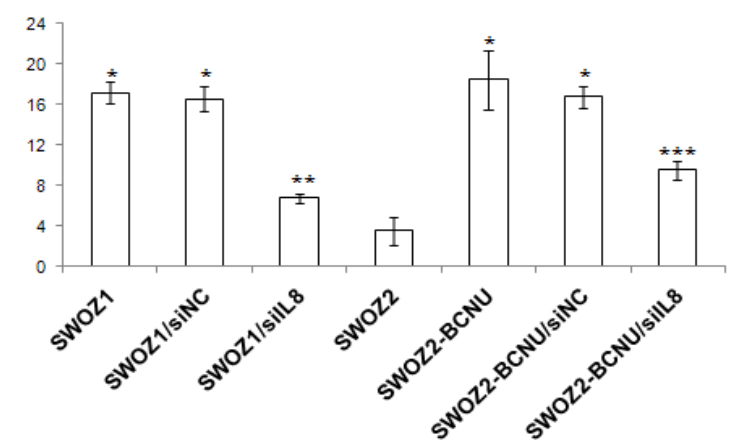

Fig. 3 The modulation of BCNU resistance after treatment with silL8-1.

(a): The growth curves of cells treated with silL8-1. The cell viability was performed by CCK-8 assay for 7 continuously days. Data are expressed as mean \pm SEM value; $n=3$.

(b): The $\mathrm{IC}_{50}$ value of all cell lines against BCNU. CCK-8 assay was used to measure the cytotoxicity of BCNU to these cells. The IC50 value, determined by the relative absorbance of CCK-8, was assessed by probit regression analysis in SPSS13.0 statistical software. Data were expressed as mean \pm SEM value; $n=3$. NC: negative control. $* P<0.05$ vs. SWOZ2; $* * P<0.05$ vs. SWOZ1/siNC; $* * * P$ $<0.05$ vs. SWOZ2-BCNU/siNC. 


\section{Discussion}

Alkylating agents, such as BCNU, are the most commonly used chemotherapeutic agents in glioma, since these agents readily cross the blood-brain barrier and have shown the most activity against malignant glioma[3, 10]. However, the development of BCNU resistant in glioma is a major clinical problem. In this study, we found that Il-8 was upregulated both in BCNU resistant cell lines SOWZ1 and SWOZ-2-BCNU compared to SWOZ2 cell line. After treatment by siRNA targeting IL-8, the BCNU resistance was partially reversed in cell lines SWOZ1 and SWOZ2-BCNU. Our results indicate that IL-8 may play an important role in the resistance of glioma to BCNU, and suggest that IL-8 may potentially serve as a therapeutic target for the treatment of glioma resistant to BNCU.

IL-8, originally described as a potent chemotactic factor for neutrophils, belongs to a superfamily of structurally related chemokines (chemotactic cytokines) that stimulate the migratory capacity of a distinct set of leukocytes, including basophils, monocytes, lymphocytes and eosinophils[11, 12]. Many of its properties, including its three-dimensional structure, receptors, signaling mechanisms and additional functions in angiogenesis, mitosis and tissue remodeling, are well-known [13]. There is an emerging literature suggesting that IL-8 expression may play an essential role in disease states, particularly tumor development and metastasis. For example, the expression of IL-8 in melanoma cells has been shown to regulate growth and metastasis in nude mice [14]. Furthermore, substantial evidence showed that IL-8 was a critical angiogenic factor in a variety of human cancers $[15,16]$. Interestingly, in this study, it was found that IL-8 was elevated in BCNU resistant glioma cell lines. Similarity result showed that IL-8 was overexpressed in a paclitaxel-resistant ovarian cancer cell line [17]. Besides, it was found that in the renal cell carcinoma, the development of sunitinib resistance was accompanied by increased expression of tumor-derived IL-8 [18]. But in another research, no appreciable increase was found in paclitaxel resistance after IL-8 was transfected into an osteosarcoma cell line [19]. The function of IL-8 in drug resistance especially in BCNU resistance needs to be further investigated.

Strategies for inhibiting IL-8 include neutralizing the protein with fully humanized antibodies against IL-8 [20, 21]. It has been demonstrated that RNAi can achieve effective, stable gene silencing in diverse biological systems and will assist in elucidating gene functions in numerous cell types including primary cells [22, 23]. Besides, it was demonstrated that the RNA interference technique can be used to efficiently down-regulate IL-8 protein expression in airway epithelial cells [24]. In this research, to explore the effect of IL-8 on BCNU resistance, siRNAs targeting IL-8 was used for inhibiting the IL-8 expression in BCNU resistant cell lines SWOZ1 and SWOZ2-BCNU. As a result, after screened for three specific siRNA sequences targeting IL-8, silL8-1, which is homologous to nt 176-194 of the IL-8 specific mRNA, was selected for further research in that the expression of IL-8 mRNA and protein was markedly decreased compared to negative control. These results demonstrated that RNAi technique may be an effective way to inhibit the IL-8 expression.

It was found that the cytotoxicity of Docetaxel, Staurosporine and Rapamycin increased significantly in IL-8 depletion cells with decreased level of Cyclin D1, Cyclin B1 and increased level of spontaneous apoptosis using RNAi technique [25]. However, few data about correlation between IL-8 expression and drug-resistance especially BCNU-resistance in glioma had been reported, and the mechanism of drug-resistance mediated by IL-8 was still unknown. In this study, it was demonstrated that accompanied by the inhibition of IL-8 protein level, the BCNU resistance was reversed for about $50 \%$ both in cell lines SWOZ1 and SWOZ2-BCNU compared to SWOZ2, 
without modulating the cellular growth. It was noted that cell lines SWOZ1 and SWOZ2 were both originated from the parental glioma cell line SWO38. Meanwhile, higher resistance to BCNU was found in cell line SWOZ1 compared to SWOZ2, which indicate that SWOZ1 was an intrinsic BCNU resistant cell line. Besides, the cell line SWOZ2-BCNU obtained from cell line SWOZ2 was an acquired BCNU resistant cell line. After the expression of IL-8 was inhibited, both of the intrinsic and acquired BCNU resistant cell lines showed more sensitive to BCNU. These results reveal that IL-8 may take as a candidate therapeutic target to reverse acquired and intrinsic BCNU-resistance in glioma.

\section{Conclusions}

Taken together, IL-8 was found to be overexpressed both in intrinsic BCNU resistant cell line SWOZ1 and acquired BCNU resistant cell line SWOZ2-BCNU compared to sensitive cell line SWOZ2. After inhibition of IL-8 expression by specific siRNA targeting IL-8, the sensitivity of both resistant cell lines SWOZ1 and SWOZ2-BCNU against BCNU was significantly increased. These data indicate that IL-8 may play an important role in glioma resistant to BCNU and IL-8 maybe a candidate therapeutic target to reverse acquired and intrinsic resistance to $\mathrm{BCNU}$ in glioma. Because of the biodiversity function of IL-8, further research should be investigated to explore the mechanism underlying this BCNU resistance mediated by IL-8 in glioma.

\section{Acknowledgments}

This research was supported by grants from National Natural Science Foundation of China (No. 81072059), National Natural Science Foundation of China for Young Scholars (No. 81101652), Science and Technology Innovation Key Project of Guangdong Higher Education Institutes (No. CXZD1110), and Natural Science Foundation of Guangdong Province (No. 10151063201000059).

\section{References}

[1] Q. Huang, Q.B. Zhang, J. Dong, Y.Y. Wu, Y.T. Shen, Y.D. Zhao, et al., Glioma stem cells are more aggressive in recurrent tumors with malignant progression than in the primary tumor, and both can be maintained long-term in vitro, BMC Cancer 8 (2008) 304.

[2] M. Westphal, D.C. Hilt, E. Bortey, P. Delavault, R. Olivares, P.C. Warnke, et al., A phase 3 trial of local chemotherapy with biodegradable carmustine (BCNU) wafers (Gliadel wafers) in patients with primary malignant glioma, Neuro. Oncology 5 (2003) 79-88.

[3] J.N. Sarkaria, G.J. Kitange, C.D. James, R. Plummer, H. Calvert, M. Weller, et al., Mechanisms of chemoresistance to alkylating agents in malignant glioma, Clinical Cancer Research 14 (2008) 2900-2908.

[4] C.L. Lin, M.H. Wang, Y.F. Qin, M. Fang, B.B. Xie, X.Y. Zhong, Differentiation of SWO-38 glioma cells induced by CDA-2 is mediated by peroxisome proliferatoractivated receptor $\gamma$, Journal of Neurooncology 95 (1) (2009) 29-36.

[5] L.X. Shi, R. Ma, R. Lu, Q. Xu, Z.F. Zhu, L. Wang, et al., Reversal effect of tyroservatide (YSV) tripeptide on multi-drug resistance in resistant human hepatocellular carcinoma cell line BEL-7402/5-FU, Cancer Letters 269 (1) (2008) 101-110.

[6] X.S. Zheng, G. Shen, X.F. Yang, W.G. Liu, Most C6 cells are cancer stem cells: Evidence from clonal and population analyses, Cancer Research 67 (2007) 3691-3697.

[7] K.J. Livak, T.D. Schmittgen, Analysis of relative gene expression data using real-time quantitative PCR and the 2 (- $\Delta \Delta \mathrm{Ct} \mathrm{C}(\mathrm{T}))$ method, Methods 25 (4) (2001) 402408.

[8] L.S. Jeffery, R. Kavitha, S. Robert, J.M. Donald, K.N. Les, A.S. Katherine, et al., Quantitative analysis of the expression of ACAT genes in human tissues by real-time PCR, Journal of Lipid Research 45 (2004) 686-696.

[9] L. Liu, Q.L. Zhang, Y. Zhang, S. Wang, Y.Q. Ding, Lentivirus-mediated silencing of Tiam1 gene influences multiple functions of a human colorectal cancer cell line, Neoplasia 8 (11) (2006) 917-924.

[10] M.K. Kang, S.K. Kang, Pharmacologic blockade of chloride channel synergistically enhances apoptosis of chemotherapeutic drug-resistant cancer stem cells, Biochemical Biophysics Research Communication 373 (4) (2008) 539-544.

[11] M. Baggiolini, B. Dewald, B. Moser, Interleukin-8 and related chemotactic cytokines-CXC and CC chemokines, Advances in Immunology 55 (1994) 97-179.

[12] N. Mukaida, Pathophysiological roles of interleukin-8/ CXCL8 in pulmonary diseases, American Journal of Physiology-Lung Cellular and Molecular Physiology 284 
(2003) L566-577.

[13] M. Baggiolini, I. Clark-Lewis, Interleukin-8, a chemotactic and inflammatory cytokine, FEBS Letters 307 (1) (1992) 97-101.

[14] R.K. Singh, M. Gutman, R. Radinsky, C.D. Bucana, I.J. Fidler, Expression of interleukin 8 correlates with the metastatic potential of human melanoma cells in nude mice, Cancer Resarch 54 (1994) 3242-3247.

[15] T.X. Xie, Z. Xia, N. Zhang, W. Gong, S. Huang, Constitutive NF-kappaB activity regulates the expression of VEGF and IL-8 and tumor angiogenesis of human glioblastoma, Oncology Reports 23 (3) (2010) 725-732.

[16] J. Heidemann, H. Ogawa, M.B. Dwinell, P. Rafiee, C. Maaser, H.R. Gockel, et al., Angiogenic effects of interleukin 8 (CXCL8) in human microvascular endothelial cells are mediated by CXCR2, Jounal of Biological Chemistry 278 (2003) 8508-8515.

[17] Z.F. Duan, J.F. Aynn, T.P. Richard, Discovery of differentially expressed genes associated with paclitaxel resistance using cDNA array technology: Analysis of Interleukin (IL) 6, IL-8, and monocyte chemotactic protein 1 in the paclitaxel resistant phenotype, Clinical Cancer Research 5 (1999) 3445-3453.

[18] H. Dan, D. Yan, Z. Ming, I.R. Brian, P. David, C.N. Qian, et al., Interleukin-8 mediates resistance to anti- angiogenic agent sunitinib in renal cell carcinoma, Cancer Research 70 (2010) 1063-1071.

[19] Z. Duan, D.E. Lamendola, R.T. Penson, K.M. Kronish, M.V. Seiden, Overexprssion of IL-6 but not IL-8 increases paclitaxel resistance of U-2OS human osteosarcoma cells, Cytokine 17 (5) (2002) 234-242.
[20] C. Ostergaard, R.V. Yieng-Kow, C.G. Larsen, N. Mukaida, K. Matsushima, T. Benfield, et al., Treatment with a monoclonal antibody to IL-8 attenuates the pleocytosis in experimental pneumococcal meningitis in rabbits when given intravenously, but not intracisternally, Clinical and Experimental Immunology 122 (2) (2000) 207-211.

[21] X.D. Yang, J.R. Corvalan, P. Wang, C.M. Roy, C.G. Davis, Fully human anti-interleukin-8 monoclonal antibodies: Potential therapeutics for the treatment of inflammatory disease states, Journal of Leukocyte Biology 66 (1999) 401-410.

[22] S.A. Stewart, D.M. Dykxhoorn, D. Palliser, H. Mizuno, E.Y. Yu, D.S. An, et al., Lentivirus -delivered stable gene silencing by RNAi in primary cells, RNA 9 (2003) 493-501.

[23] S.L. Huang, Y. Wu, H. Yu, P. Zhang, X.Q. Zhang, L. Ying, et al., Inhibition of Bcl-2 expression by a novel tumor-specific RNA interference system increases chemosensitivity to 5-fluorouracil in Hela cells, Acta Pharmacologica Sinica 27 (2) (2006) 242-248.

[24] H.B. Cao, A. Wang, B. Martin, D.R. Koehler, P.L. Zeitlin, A.K. Tanawell, et al., Down-regulation of IL-8 expression in human airway epithelial cells through helper-dependent adenoviral-mediated RNA interference, Cell Research 15 (2) (2005) 111-119.

[25] K.S. Rajendra, L.L. Bal, Depletion of intrinsic expression of Interleukin-8 in prostate cancer cells causes cell cycle arrest, spontaneous apoptosis and increases the efficacy of chemotherapeutic drugs, Molecular Cancer 8 (2009) 57-72. 